\title{
Fratura do processo transverso da vértebra lombar: valor diagnóstico
}

\author{
Carlos Umberto Pereira', Eldon Bezerra Silva Junior², Liani Patrícia Andrade Santos² \\ Departamento de Medicina da Universidade Federal de Sergipe (UFS), Aracaju, Sergipe.
}

\section{RESUMO}

As fraturas isoladas do processo transverso são consideradas lesões menores. Em geral, são causadas por trauma direto ou avulsão resultante da contração muscular. Radiografias simples são pouco sensíveis para a detecção dessas fraturas, portanto a verdadeira frequência dessas fraturas pode ser subestimada. Com o advento da tomografia computadorizada (TC) helicoidal, tem aumentado à frequência dessa lesão. GS, 53 anos, masculino. Vítima de sequestro e colocado no porta-malas do carro. No setor de emergência, apresentou queixa de dores generalizadas, mais intensa na região lombar. Exame neurológico: normal. Rx simples de abdome: normal. Tomografia da coluna lombar: fratura do processo transverso esquerdo de L5. Tratamento conservador, com evolução satisfatória. Apesar de ser considerada um traumatismo leve, a fratura do processo transverso da vértebra lombar ocorre como resultado de forças intensas. Geralmente, está associado com lesão de vísceras abdominais ou geniturinárias. Seu diagnóstico tem sido feito por meio de exame de TC helicoidal. Seu tratamento inicial é conservador. Fraturas do processo transverso das vértebras lombares cada vez mais têm sido diagnosticadas. Existe associação com lesões de vísceras abdominais e geniturinárias. O tratamento inicial é conservador e cursa com bom prognóstico.

\section{PALAVRAS-CHAVE}

Traumatismos da coluna vertebral/diagnóstico, traumatismos da coluna vertebral/terapia, traumatismos da medula espinhal/diagnóstico.

\section{ABSTRACT}

Lumbar vertebra transverse process fracture: diagnostic value

Isolated fractures of the transverse process are considered minor injuries usually are caused by direct trauma or avulsion resulting from muscle contraction. Plain radiographs are insensitive for the detection of these fractures, so the true frequency of these fractures may be underestimated. With the advent of helical computed tomography, has increased the frequency of this injury. GS, age 53, male. Victim of kidnapping and placed in the trunk of the car. In the emergency room complaining of aches, more severe in the lumbar region. Neurological examination: normal. Simple X-ray of the abdomen: normal. Tomography of the lumbar spine: fracture of the left transverse process of $L 5$. Conservative treatment with a favorable outcome. Although considered a mild injury, the fracture of the transverse process of lumbar vertebra occurs as a result of strong forces. It is usually associated with injury to abdominal viscera or genitourinary. Its diagnosis has been made by helical CT. His initial treatment is conservative. Fracture of transverse process of lumbar vertebrae has been increasingly diagnosed. There is an association with lesions of the abdominal viscera and genitourinary. Initial treatment is conservative and runs with good prognosis.

\section{KEYWORDS}

Spinal injuries/diagnosis, spinal injuries/therapy, spinal cord injuries/diagnosis.

\section{Introdução}

A fratura isolada do processo transverso tem sido mencionada como lesão menor, geralmente sendo causada por trauma direto ou avulsão resultante da contração muscular..$^{1-4}$ No entanto, as grandes forças necessárias para causá-la também podem resultar em lesão abdominal, torácica ou geniturinária associada. ${ }^{4-7}$ Por isso, faz-se necessária uma avaliação clínica e radiológica detalhada. ${ }^{8}$ As radiografias convencionais são insensíveis para a deteç̧ão dessa lesão, portanto a verdadeira frequência das fraturas do processo transverso é subestimada. Com o uso rotineiro nos serviços de emergência da tomografia computadorizada (TC)

1 Professor doutor adjunto do Departamento de Medicina da Universidade Federal de Sergipe (UFS), Serviço de Neurocirurgia do Hospital de Urgência de Sergipe (HUSE), Aracaju, Sergipe, Brasil.

2 Doutorando de Medicina da UFS, Aracaju, Sergipe, Brasil. 
helicoidal, a frequência desse achado tem aumentado. Essas lesões são consideradas estáveis e seu tratamento tem sido inicialmente conservador. ${ }^{9}$

Os autores relatam um caso de fratura isolada do processo transverso da quinta vértebra lombar. São analisados os meios de diagnóstico e conduta.

\section{Relato do caso}

Paciente, 61 anos, masculino, motorista. Vítima de assalto, foi colocado no porta-malas do automóvel. Deu entrada no setor de emergência com dores generalizadas, porém mais intensas na região lombar. Desperto e eupneico. Exame neurológico: normal. Radiografia simples de abdome: normal. TC de coluna lombar: fratura do processo transverso esquerdo de L5 (Figuras 1A, 1B e 2). Submetido a tratamento conservador (repouso e analgésicos). Recebeu alta médica sem queixas neurológicas.
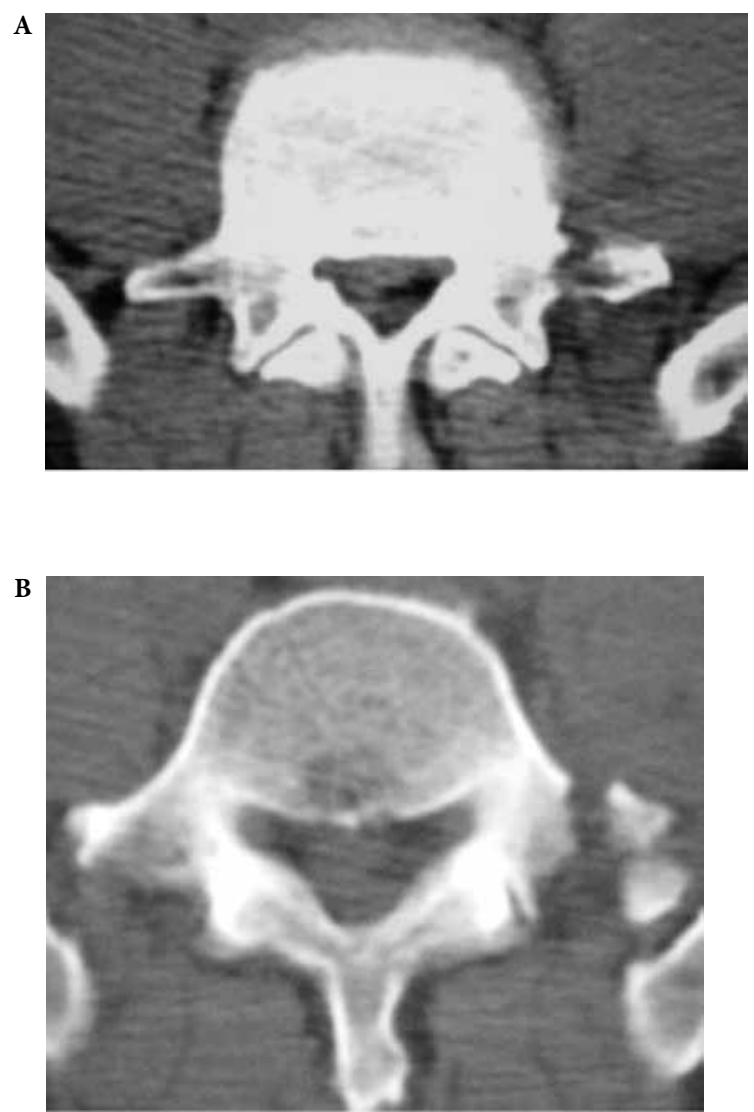

Figuras $1 A$ e $1 B$ - TC de coluna lombar apresentando fratura do processo transverso esquerdo de L5.

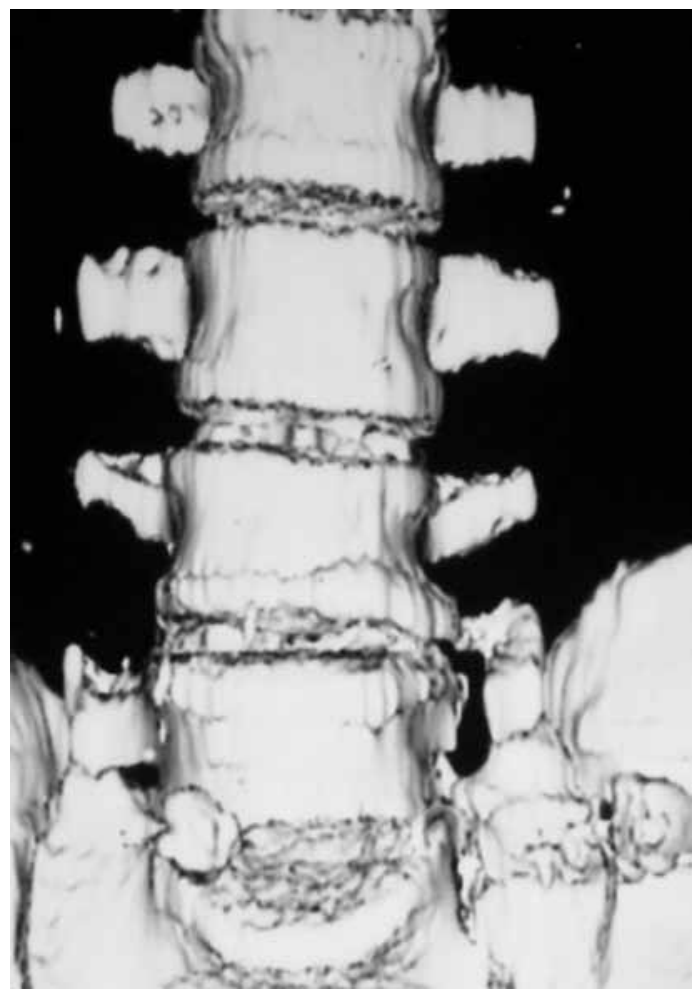

Figura 2 - TC helicoidal da coluna lombar demonstrando fratura isolada do processo transverso esquerdo de L5.

\section{Discussão}

As fraturas dos processos transversos das vértebras lombares são consideradas traumatismos relativamente leves, mas ocorrem como resultado de forças maiores. Embora essas fraturas sejam frequentemente múltiplas, são, todavia, consideradas leves e estáveis. ${ }^{2,6}$ Elas podem resultar de trauma contuso direto, forças de flexão-extensão laterais violentas, avulsão do músculo psoas ou fraturas de Malgaigne da pelve. ${ }^{3,4,7,10}$ No entanto, as grandes forças necessárias para causá-las também podem resultar em lesão abdominal, torácica, geniturinária, ossos longos, crânio e medula espinhal associadas ${ }^{3,7,11}$, porém pode ocorrer em ausência de lesões vertebrais e viscerais, ${ }^{3,4,12}$ fato esse observado em nosso caso.

A detecção dessas fraturas em radiografias pode predizer a presença de lesões adicionais no paciente politraumatizado. ${ }^{6,7,11}$ Classicamente, as radiografias simples da coluna lombar são utilizadas para a avaliação desses pacientes com trauma contuso, no entanto é relativamente insensível na detecção das fraturas de processo transverso, bem como de lesões viscerais associadas. ${ }^{3,12,13}$ Portanto, a verdadeira frequência das fraturas do processo transverso das vértebras lombares pode ser subestimada, por meio dos achados radiográficos convencionais isolados. ${ }^{7}$ Essa insensibilidade das radiografias convencionais, especialmente na definição 
do trauma agudo, pode ser justificada devido à presença de gases intestinais sobrejacentes, o que faz a avaliação completa da extensão das fraturas e lesões associadas não ser possível. ${ }^{4,7,12,13} \mathrm{Em}$ nosso caso, não foi diagnosticada a presença de fratura do processo transverso esquerdo da L5 na radiografia simples de abdome.

O exame de TC tem sido uma técnica cada vez mais importante na avaliação dos pacientes com trauma abdominal. ${ }^{7}$ A TC possui um importante papel na avaliação das fraturas da coluna vertebral, permitindo a avaliação de todo o anel ósseo que circunda o canal vertebral, em especial a parede posterior do corpo vertebral, identificando áreas de compressão das estruturas nervosas e quantificando o grau de compressão. As estruturas posteriores das vértebras podem também ser mais bem visualizadas pela TC, e a reconstrução tridimensional por tal técnica ampliou suas vantagens, possibilitando a visualização de deformidades e deslocamentos no plano axial e de fraturas horizontais a partir da vértebra. Com o uso generalizado da TC de corpo inteiro em pacientes com trauma, as fraturas isoladas de processos transversos têm sido cada vez mais reconhecidas. ${ }^{3,7,12,14}$ Em nosso paciente, o diagnóstico foi realizado após realização do exame de TC. A RM possibilita a observação e a delimitação dos tecidos moles e de suas lesões, principalmente ligamentos, disco intervertebral e medula espinhal. Esse exame permite delimitar o nível da lesão medular e a diferenciação entre hematoma e edema, que apresenta importância na conduta e no prognóstico.

Os principais objetivos do tratamento do TRM são: restauração da anatomia e das condições fisiológicas do segmento vertebral lesado, restabelecimento da função máxima do paciente e da estabilidade do segmento vertebral lesado, presença de dor residual mínima, recuperação do déficit neurológico, prevenção de incapacidade futura e de dor residual, início precoce da reabilitação e retorno às atividades profissionais.

As fraturas isoladas do processo transverso, quando não são associadas a déficits neurológicos ou instabilidade estrutural, devem ser tratadas de maneira conservadora. ${ }^{14}$ No entanto, essa conduta só deve ser adotada após a exclusão de instabilidade da coluna vertebral e de lesões associadas. Nosso paciente foi submetido a tratamento conservador e apresentou resultado excelente.

Assim, na presença de fratura isolada de processo transverso lombar, deve-se investigar a presença de lesões de vísceras abdominais e geniturinária, para evitar maiores danos nesses pacientes.

\section{Referências}

1. Barker PJ, Urquhart DM, Story IH, Fahrer M, Briggs CA. The middle layer of lumbar fascia and attachments to lumbar transverse processes: implications for segmental control and fracture. Eur Spine J. 2007;16(12):2232-7.

2. Gilsanz V, Miranda J, Cleveland R, Willi U. Scoliosis secondary to fractures of the transverse processes of lumbar vertebrae. Radiology. 1980;134(3):627-9.

3. Krueger MA, Green DA, Hoyt D, Garfin SR. Overlooked spine injuries associated with lumbar transverse process fractures. Clin Orthop Relat Res. 1996;(327):191-5.

4. Miller CD, Blyth P, Civil ID. Lumbar transverse process fractures: a sentinel marker of abdominal organ injuries. Injury. 2000;31(10):773-6.

5. Dağlar B, Bayrakçi K, Taşbaş BA, Dağlar G, Ozdemir G, Delialioğlu OM, et al. [Importance of lumbar vertebra transverse process fractures in multitrauma patients who had undergone emergent laparotomy]. Ulus Travma Acil Cerrahi Derg. 2005;11(1):58-63.

6. Denis $F$. The three column spine and its significance in the classification of acute thoracolumbar spinal injuries. Spine (Phila Pa 1976). 1983;8(8):817-31.

7. Patten RM, Gunberg SR, Brandenburger DK. Frequency and importance of transverse process fractures in the lumbar vertebrae at helical abdominal CT in patients with trauma. Radiology. 2000;215(3):831-4.

8. Homnick A, Lavery R, Nicastro O, Livingston DH, Hauser CJ. Isolated thoracolumbar transverse process fractures: call physical therapy, not spine. J Trauma. 2007;63(6):1292-5.

9. Bali K, Kumar V, Krishnan V, Meena D, Rawall S. Multiple lumbar transverse process stress fractures as a cause of chronic low back ache in a young fast bowler - a case report. Sports Med Arthrosc Rehabil Ther Technol. 2011;3(1):8.

10. Brynin R, Gardiner L. Missed lumbar transverse process fractures in a high school football player. J Manipulative Physiol Ther. 2001;24(2):123-6.

11. Sturm JT, Perry JF Jr. Injuries associated with fractures of the transverse processes of the thoracic and lumbar vertebrae. J Trauma. 1984;24(7):597-9.

12. Agrawal A, Srivastava $S$, Kakani A. Isolated transverse process fracture of the lumbar vertebrae. J Emerg Trauma Shock. 2009;2(3):217-8.

13. Gestring ML, Gracias VH, Feliciano MA, Reilly PM, Shapiro $\mathrm{MB}$, Johnson JW, et al. Evaluation of the lower spine after blunt trauma using abdominal computed tomographic scanning supplemented with lateral scanograms. J Trauma. 2002;53(1):9-14.

14. Bradley LH, Paullus WC, Howe J, Litofsky NS. Isolated transverse process fractures: spine service management not needed. J Trauma. 2008;65(4):832-6.

Endereço para correspondência

Carlos Umberto Pereira

Av. Augusto Maynard, 245/404, Bairro São José

49015-380 - Aracaju, Sergipe

E-mail : umberto@infonet.com.br

\section{Conflito de interesses}

Os autores declaram inexistência de conflito de interesses no presente trabalho. 